\title{
Immunologic Aspects of Perioperative Nutrition
}

\author{
L. Andersen ${ }^{1, *}$, M. Rasmussen ${ }^{2}$ and R. Beier-Holgersen ${ }^{3}$ \\ ${ }^{1}$ Department of Gastrointestinal Surgery, Sjaellands University Hospital Koege, Denmark \\ ${ }^{2}$ Department of Gastrointestinal Surgery, Holbaek Hospital, Denmark \\ ${ }^{3}$ Department of Gastrointestinal Surgery, Nordsjaellands Hospital, Denmark
}

\begin{abstract}
Nutrition has proven to be of great importance for the postoperative clinical outcome. Several studies have shown that infectious complications in the surgical patient, are reduced by pre- or postoperative nourishment. We discuss cellular immunity in relation to both enteral and parenteral nutrition and present an updated literature study of current evidence. The aim of this paper is to give an overview of studies, that compare different immunological parameters in the surgical patient being nourished either enterally or parenterally.
\end{abstract}

Keywords: Parenteral nutrition, enteral nutrition, cellular immune response, surgery.

\section{INTRODUCTION}

During the postoperative period, nutrition has proven to be of great importance for the postoperative clinical outcome. Several studies have shown that by selective nourishing of patients undergoing surgery, infectious complications are reduced [1-3].

However, the optimal pathway (either preoperatively or postoperatively) to nourish patients is unknown. Some studies suggest that nourishing patients parenterally does not significantly reduce infections postoperatively $[1,4,5]$.

Studies directly comparing enteral and parenteral postoperative nutrition have shown that enteral nutrition significantly reduced infectious complications compared with parenteral nutrition [5]. The immunological reason for this presumed effect is not well understood.

The aim of this paper is to give an updated overview of studies, that compare specific immunological parameters of patients being nourished enterally and parenterally, pre- and postoperatively.

\section{METHODS}

We conducted a literature search in PubMed by 2 independent investigators focusing on cellular immune response parameters, the surgical patient and the perioperative period. The search was divided into enteral and parenteral nutrition.

*Address correspondence to this author at the Department of Gastrointestinal Surgery, Sjaellands University Hospital Koege, Lykkebaekvej 1, 4600 Koege, Denmark; Tel: +4547323011; Fax: +4556631569;

E-mail: lene_andersen_@hotmail.com
The primary aim was to investigate evidence for changes in immunologic parameters dependent on different routes of nutrition (enteral or parenteral). $\mathrm{MeSH}$ terms used were: "enteral nutrition", "parenteral nutrition", "cellular immune response", "surgery".

\section{Cellular Immunity, Terminology}

Cellular immunity is divided into the innate (nonspecific) immune system consisting of macrophages and other cells doing phagocytosis, and the adaptive (specific) immune system composed of lymphocytes.

In literature, measurements of cellular immunity activity are often described according to the percentage of different "clusters of differentiation" (CD) eg. CD3+, CD4+, CD3- present in blood (Table 1). An alternative presentation is by subtype in the majorhistocompatibility complex molecule (MHC II). MHC II is divided in different human leucocyte antigens, ("HLAs") such as HLA-DR.

Table 1: Common Immunologic Parameters Expressing Cellular Immune Activity

\begin{tabular}{|c|c|}
\hline Name & Immune function \\
\hline \hline CD3+ & Pan T-cell \\
\hline CD4+ & Helper cell \\
\hline CD8+ & Cytotoxic cell \\
\hline CD3- & NK cell \\
\hline CD16+ & NK cell \\
\hline CD56+ & NK cell \\
\hline CD69+ & Activated T-cell and NK cell \\
\hline IL-6 & Proinflammatory cytokine, B-cell activator \\
\hline IL-10 & Anti-inflammatory cytokine, T-cell production \\
\hline
\end{tabular}


In much of the literature, the number of cell changes over time is expressed as a change in percentage. Unfortunately, many authors do not indicate the actual number of lymphocytes. A direct comparison of lymphocyte count and the immunological markers change over time is therefore often impossible. To compare percentage values at different times without an actual lymphocyte count in relation to these subpopulations is not possible. However, comparison is possible by usage of ratios, e.g. the CD4/CD8 ratio.

\section{Enteral Nutrition and Cellular Immunity}

In a randomized controlled trial including 60 patients, Beier-Holgersen and Brandstrup [6] compared 2 different postoperative enteral nutritional regimes (food vs. no food) and immunologic response. A significantly larger number of lymphocytes, monocytes, NK cells and also activated T cells (CD69+), were found in the food group when measured postoperatively compared to the no food group. HLADR activated cells significantly increased from the third post-operative day onwards, compared to the "no food" group. The results indicate that cellular immunity activity was significantly higher in the food group.

Sultan et al. [7] compared a pre- and postoperative enteral nutrition regime in a group receiving postoperative enteral feeding (based solely on the clinical consultants' preference). The patients were randomized to enteral nutrition with or without supplemented omega-3 fatty acids. 195 patients undergoing oesophago-gastric cancer surgery were included. The results did not show any significant effect on either immune parameters measured as HLA-DR expression in leucocytes or on clinical outcome.

In a prospective study $\mathrm{Li}$ et al. [8] compared postoperative enteral and parenteral nutrition in 400 patients after radical gastrectomy. The enteral nutrition group regained preoperative levels of CD4, CD4/CD8 and NK cells faster postoperatively compared to the parenteral nutrition group were levels were significantly lower. There was no difference in clinical outcome.

Takagi $\mathrm{K}$ et al. [9] compared perioperative enteral and parenteral nutrition in a retrospective study in 29 patients undergoing esophagectomy. IL-6, IL-10 as well as endotoxins (part of the cell wall of gram-negative bacteria) were measured. The postoperative endotoxin concentration in sera was significantly lower in the group receiving enteral nutrition. Based on these results the authors suggest that perioperative enteral nutrition provides better regulation of inflammatory cytokine response and may contribute to less immunosuppression after major surgery then parenteral nutrition.

Suzuki D et al. [10] studied 30 patients undergoing pancreatico-duodenectomy, in a prospective study. 3 groups received enteral nutrition pre- and postoperatively, enteral nutrition postoperatively or parenteral nutrition postoperatively. T cell and NK cell activity were significantly higher in the group receiving enteral nutrition pre- and postoperatively compared to the other groups, as well as other measured immunologic parameters. The authors concluded that perioperative nutrition reduces the rate of postoperative infections.

\section{Parenteral Nutrition and Cellular Immunity}

Furukawa et al. [11] randomized 36 patients undergoing abdominal surgery to parenteral nutrition with or without added soybean emulsion. Immunologic parameters measured was IL- 6 and T-cell/NK cell proliferation. The addition of soybean emulsion increased the stress response and repressed the cellular immune response in already severely stressed patients. The study did not include a group receiving "no food" compared to parenteral nutrition.

In a prospective randomized study by Dionigi $\mathrm{P} D R$ et al. [12], 15 malnourished patients undergoing operations for upper $\mathrm{Gl}$-cancer were randomized to receive parenteral nutrition with either intralipid or glucose postoperatively. The results showed that Tand B-lymphocyte count, chemotaxis and granulocyte adherence were depressed in the group recieving parenteral nutrition with added glucose. The authors concluded that postoperative infections were less severe in patients receiving intralipid.

Long et al. [13] compared 60 patients undergoing esophageal cancer surgery in a prospective study, who received parenteral nutrition with or without omega3polyunsaturated fatty acids ( $\omega 3$-PUFAs). Procalcitonin, an inflammatory marker and CD4+/CD8+ ratios were determined perioperatively. In the group receiving $\omega 3$ PUFAs the CD4+/CD8+ ratio were higher and procalcitonin lower. The authors thus suggest that $\omega 3-$ PUFAs can reduce inflammation and improve immune function postoperatively.

A prospective randomized (single blinded) study by Yao et al. [14] included 61 patients undergoing surgery 
for bowel obstruction due to chronic radiation enteritis. The patients were randomized to parenteral nutrition alone or supplemented with glutamine dipeptide. Outcomes were serum glutamine level and the CD4/CD8-positive T-lymphocytes ratio. The group receiving glutamine was less prone to developing fever, but no other clinical difference were found. The CD4/CD8-positive T-lymphocytes ratios rose in both groups, however with significant higher ratio measured in the glutamine supplemented group.

A prospective single blinded trial by JM Asprer et al. [15] randomized 34 malnourished patients undergoing elective abdominal surgery, to preoperative parenteral supplementation with or without added glutamine dipeptide. Primary outcomes were several different "immune indices" measured as change in cell count. In the group receiving glutamine supplementation an increase in immune cells were found compared to no change in the group receiving only parenteral nutrition. The clinical outcome between groups were similar and the effect on immune cells were not sustained after glutamine dipeptide supplementation stopped.

\section{DISCUSSION}

The literature concerning effect of enteral or parenteral nutrition on cellular immunologic parameters is sparse. Studies include few patients, conclusions are drawn on different immune cell lines and seldom with measurable clinical difference. Study designs vary considerable e.g. feeding routes and nutritional components. A specific connection between varying immunologic parameters and clinical outcome is yet to be identified.

Many studies show a measurable percentage difference between nutritional intervention by the usage of subpopulations of lymphocytes, without known lymphocyte count. This does not describe the immunological aspects correctly $(15,16,18,19,20)$.

For the patient undergoing major surgery, nutritional status betters clinical outcome [1-3] but the immunologic parameters that correspond to clinical results and nutritional regime is unknown. There seems to be a larger effect of enteral nutrition on immunological parameters then parenteral nutrition $(12$, $13,14)$.

The inherent challenge of supplying sufficient nutrition to patients undergoing surgery especially to the $\mathrm{Gl}$ tract also warrants further research because nutritional absorption naturally depends on mucosal integrity and gastrointestinal continuity [16].

Further studies must identify both the specific immunologic parameters that correlate to relevant clinical outcome and measure effect in different routes of feeding.

\section{REFERENCES}

[1] Mochizuki H, Togo S, Tanaka K, Endo I, Shimada H. Early enteral nutrition after hepatectomy to prevent postoperative infection. Hepatogastroenterology 2000; 47(35): 1407-1410.

[2] Okamoto K, Fukatsu K, Hashiguchi $Y$, Ueno $H$, Shinto E, Moriya T, Saitoh D, Yamamoto J, Hase K. Lack of preoperative enteral nutrition reduces gut-associated lymphoid cell numbers in colon cancer patients: a possible mechanism underlying increased postoperative infectious complications during parenteral nutrition. Ann Surg 2013; 258(6): 1059-1064.

\section{http://dx.doi.org/10.1097/SLA.0b013e31827a0e05}

[3] Beier-Holgersen R, Boesby S. Influence of postoperative enteral nutrition on postsurgical infections. Gut 1996; 39(6): 833-835.

http://dx.doi.org/10.1136/gut.39.6.833

[4] Pham KN, Schwartz LW, Garg T, Langenstroer P, Guralnick $\mathrm{ML}$, See WA, O'Connor RC. Immediate total parenteral nutrition after radical cystectomy and urinary diversion. WMJ 2014; 113(1): 20-23.

[5] Bozzetti F, Braga M, Gianotti L, Gavazzi C, Mariani L. Postoperative enteral versus parenteral nutrition in malnourished patients with gastrointestinal cancer: a randomised multicentre trial. Lancet 2001; 358(9292): 14871492.

http://dx.doi.org/10.1016/S0140-6736(01)06578-3

[6] Beier-Holgersen R, Brandstrup B. Influence of postoperative enteral nutrition on cellular immunity. A random doubleblinded placebo controlled clinical trial. Int J Colorectal Dis 2012; 27(4): 513-520. http://dx.doi.org/10.1007/s00384-011-1335-0

[7] Sultan J, Griffin SM, Di Franco F, Kirby JA, Shenton BK, Seal CJ, Davis P, Viswanath YK, Preston SR, Hayes N. Randomized clinical trial of omega- 3 fatty acid-supplemented enteral nutrition versus standard enteral nutrition in patients undergoing oesophagogastric cancer surgery. $\mathrm{Br} \mathrm{J}$ Surg 2012; 99(3): 346-355. http://dx.doi.org/10.1002/bjs.7799

[8] Li B, Liu HY, Guo SH, Sun P, Gong FM, Jia BQ. The postoperative clinical outcomes and safety of early enteral nutrition in operated gastric cancer patients. J BUON 2015; 20(2): 468-472.

http://dx.doi.org/10.1016/j.bone.2015.08.017

[9] Takagi K, Yamamori H, Toyoda Y, Nakajima N, Tashiro T. Modulating effects of the feeding route on stress response and endotoxin translocation in severely stressed patients receiving thoracic esophagectomy. Nutrition 2000; 16(5): 355-360. http://dx.doi.org/10.1016/S0899-9007(00)00231-8

[10] Suzuki D, Furukawa K, Kimura F, Shimizu H, Yoshidome $H$, Ohtsuka M, Kato A, Yoshitomi H, Miyazaki M. Effects of perioperative immunonutrition on cell-mediated immunity, $\mathrm{T}$ helper type 1 (Th1)/Th2 differentiation, and Th17 response after pancreaticoduodenectomy. Surgery 2010; 148(3): 573581.

http://dx.doi.org/10.1016/j.surg.2010.01.017

[11] Furukawa K, Yamamori H, Takagi K, Hayashi N, Suzuki R, Nakajima N, Tashiro T. Influences of soybean oil emulsion on 
stress response and cell-mediated immune function in moderately or severely stressed patients. Nutrition 2002; 18(3): 235-240. http://dx.doi.org/10.1016/S0899-9007(01)00784-5

[12] Dionigi P DR, Prati U, Pavesi F, Jemos V, Nazari S. Effect of intralipid on some immunological parameters and leukocyte functions in patients with esophageal and gastric cancer. Clin Nutr 1985(4): 229-234.

http://dx.doi.org/10.1016/0261-5614(85)90008-1

[13] Long $H$, Yang $H$, Lin $Y$, Situ D, Liu W. Fish oil-supplemented parenteral nutrition in patients following esophageal cancer surgery: effect on inflammation and immune function. Nutr Cancer 2013; 65(1): 71-75.

http://dx.doi.org/10.1080/01635581.2013.741761
[14] Yao D, Zheng L, Wang J, Guo M, Yin J, Li Y. Perioperative Alanyl-Glutamine-Supplemented Parenteral Nutrition in Chronic Radiation Enteritis Patients With Surgical Intestinal Obstruction: A Prospective, Randomized, Controlled Study. Nutr Clin Pract 2015.

[15] Asprer JM, et al. Effect on immune indices of preoperative intravenous glutamine dipeptide supplementation in malnourished abdominal surgery patients in the preoperative and postoperative periods. Nutrition 2009; 25(9): 920-5. http://dx.doi.org/10.1016/j.nut.2009.01.014

[16] Mukhopadhyay A, et al. Can Postoperative Nutrition be Favourably Maintained by Oral Diet in Patients with Emergency Temporary lleostomy? A Tertiary Hospital Based Study. J Clin Diagn Res 2015; 9(12): PC04-8. http://dx.doi.org/10.7860/jcdr/2015/15220.6879 